\title{
3D Imaging Using HAADF-STEM and HRTEM Atomic Electron Tomography
}

Colin Ophus ${ }^{1 *}$, David Ren ${ }^{2}$, Jihan Zhou ${ }^{3}$, Hannah Devyldere ${ }^{1}$, Michael Chen ${ }^{2}$, Philipp M Pelz ${ }^{4}$, Peter Ercius $^{1}$, Jianwei Miao ${ }^{3}, \mathrm{MC} \mathrm{Scott}^{1,4}$ and Laura Waller $^{2}$

1. NCEM, Molecular Foundry, Lawrence Berkeley National Laboratory, Berkeley, USA.

2. Dept. of Electrical Engineering and Computer Sciences, Univ. of California Berkeley, Berkeley, USA.

3. Dept. of Physics and Astronomy, Univ. of California Los Angeles, Los Angeles, USA.

4. Dept. of Materials Science and Engineering, Univ. of California Berkeley, Berkeley, USA.

* Corresponding author: cophus@gmail.com

The ability to image samples at atomic resolution using transmission electron microscopy (TEM) is a very powerful tool for materials science. By using aberration-corrected high angle annular dark fieldscanning transmission electron microscopy (HAADF-STEM) to record images over a large range of tilt angles, we can reconstruct a sample in 3D at atomic resolution using computational imaging methods. This method, called atomic electron tomography (AET) can also determine the species of each atom [1]. Recently, we have added the dimension of time to these experiments to produce a series of AET reconstructions to measure the atomic-scale evolution of a sample during ex-situ annealing [2]. Figure 1a shows three AET reconstructions of a platinum-iron nanoparticle at different annealing times. FePt nanoparticles are scientifically interesting because of their potential use as magnetic storage media. The magnetic FePt phase has an L1 0 structure composed of alternating Fe and Pt planes. During annealing, several $\mathrm{L}_{0}$ domains nucleate and grow from the surface of the initially disordered FePt nanoparticle, measured using a local order parameter in Figure 1b. The population statistics are shown in Figure 1c. Time-resolved AET is a powerful new method to study nucleation and growth on the atomic scale.

HAADF-STEM produces nearly linear 2D projections of the sample structure for thin samples with medium or high atomic numbers which are ideal for tomography experiments. However, HAADF imaging is insensitive to weakly-scattering elements such as $\mathrm{C}$ or $\mathrm{O}$, and is relatively dose-inefficient [3]. Imaging these classes of samples will require a phase contrast imaging method. We have chosen to use plane-wave focal series high-resolution (HRTEM) tilt series to perform tomography experiments. The experimental geometry is shown in Figure 2a. A multislice simulation of two tilt angles from a sample composed of a crystalline $\mathrm{Si}$ core and an amorphous $\mathrm{SiO}_{2}$ sample is plotted in Figure $2 \mathrm{~b}$. As this simulation shows, the measured image intensity is a highly nonlinear function of the sample's projected potential, and when using a low electron dose is quite noisy. To address these issues, we have developed a 3D reconstruction method that accurately models multiple scattering of the electron beam, with simulation results shown in Figure 2c [4]. Phase contrast AET experiments are in progress - an example of focal series reconstructions recorded at different tilts of an oxidized tungsten tip is shown in Figure 3. Note the strongly nonlinear phase contrast, which varies rapidly with sample tilt [5].

References:

[1] Y Yang et al., Nature 542 (2018), p. 75.

[2] J Zhou et al., arXiv:1807.10709 (2018).

[3] C Ophus et al., Nature Communications 7 (2016), p. 10719.

[4] D Ren et al., arXiv:1807.03886 (2018).

[5] Work at the Molecular Foundry was supported by the Office of Science, Office of Basic Energy

Sciences, of the U.S. Department of Energy under Contract No. DE-AC02-05CH11231. C Ophus acknowledges support of the DoE Early Career Research Program. 

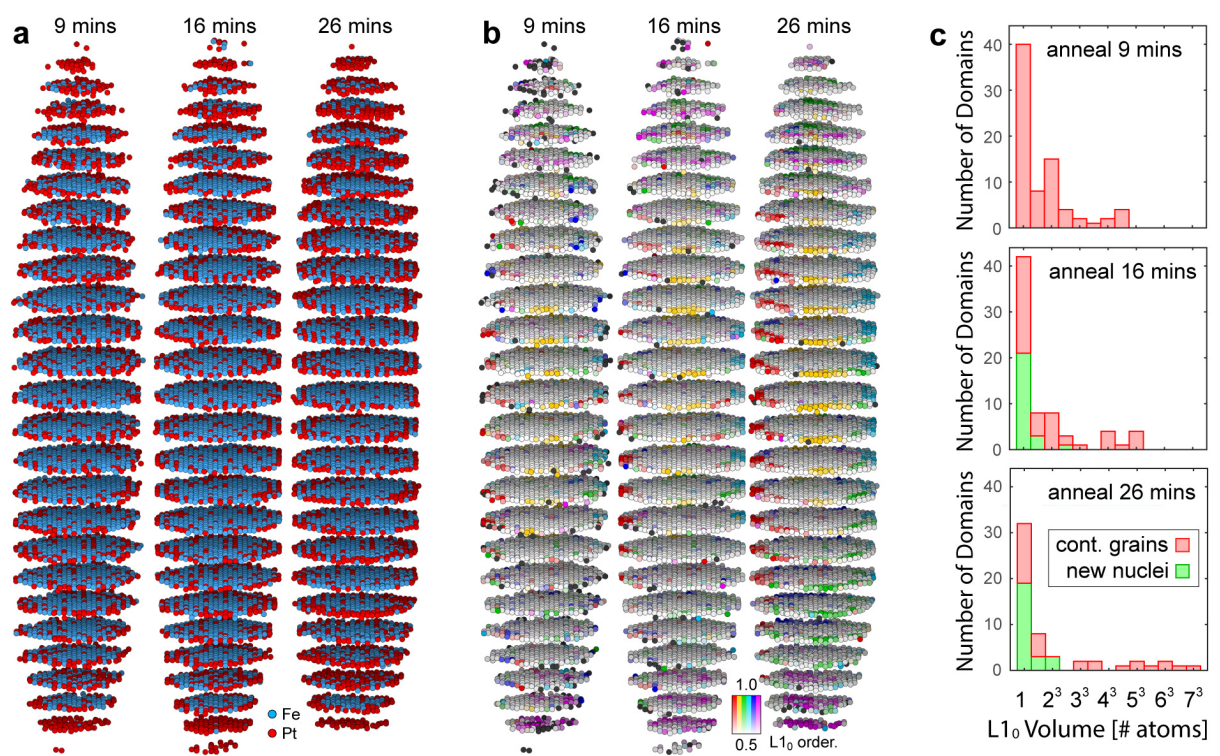

Figure 1. Atomic electron tomography of an FePt nanoparticle, (a) position and species measured at different annealing times. (b) Local $\mathrm{L} 1_{0}$ chemical ordering for the three annealing times. (c) Number and size of $\mathrm{L} 1_{0}$ domains identified at each time point, including newly formed nuclei. Adapted from Ref. [2].

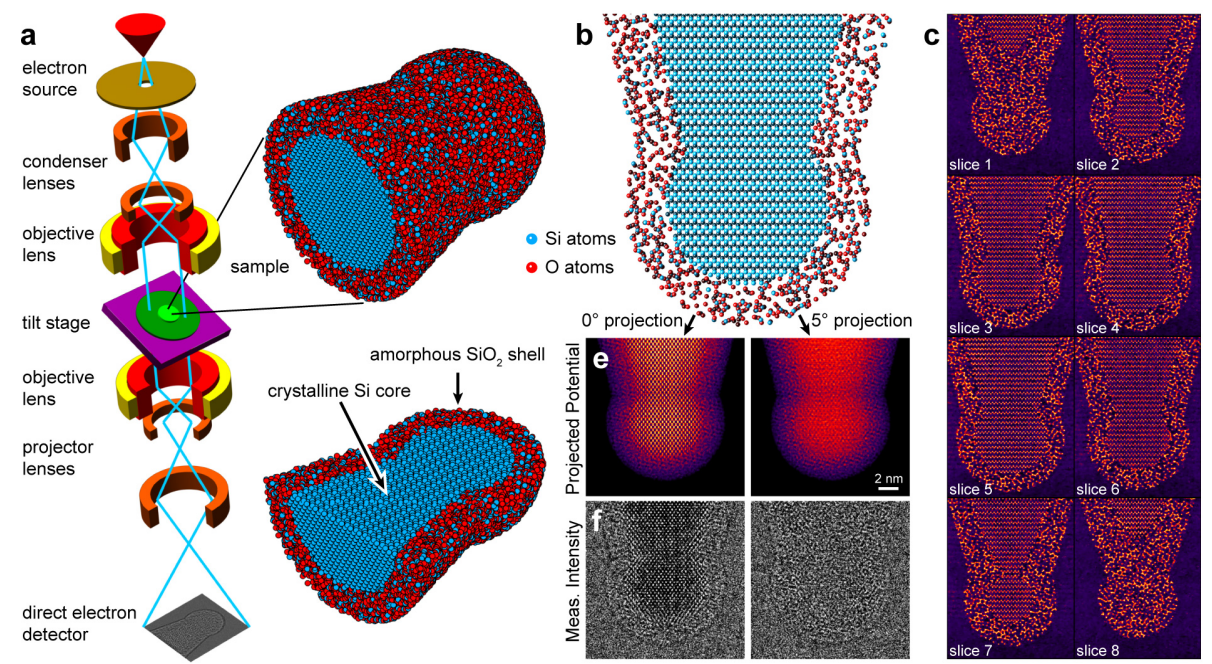

Figure 2. Phase contrast AET. (a) Geometry of a tilt-defocus HRTEM experiment. (b) Multislice simulation of a $\mathrm{Si}_{-} \mathrm{SiO}_{2}$ core-shell tip. (c) Slices from the reconstructed 3D volume, from Ref. [4].
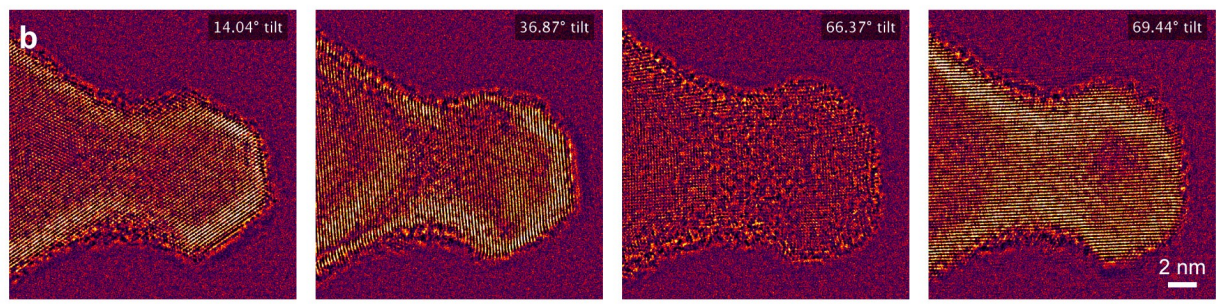

Figure 3. Phase images of experimental focal series reconstructions of a tip composed of a tungsten core and tungsten oxide shell, recorded at various sample tilts. 